\title{
Optimization of Free Space Optical Wireless Network for Cellular Backhauling
}

\author{
Yuan Li, Nikolaos Pappas, Vangelis Angelakis, Michal Pioro and Di Yuan
}

Linköping University Post Print

Tweet

N.B.: When citing this work, cite the original article.

Yuan Li, Nikolaos Pappas, Vangelis Angelakis, Michal Pioro and Di Yuan, Optimization of Free Space Optical Wireless Network for Cellular Backhauling, 2015, IEEE Journal on Selected Areas in Communications, (33), 9, 1841-1854.

$\underline{\text { http://dx.doi.org/10.1109/JSAC.2015.2432518 }}$

C2015 IEEE. Personal use of this material is permitted. However, permission to reprint/republish this material for advertising or promotional purposes or for creating new collective works for resale or redistribution to servers or lists, or to reuse any copyrighted component of this work in other works must be obtained from the IEEE.

\section{http://ieeexplore.ieee.org/}

Postprint available at: Linköping University Electronic Press

http://urn.kb.se/resolve?urn=urn:nbn:se:liu:diva-121428 


\title{
Optimization of Free Space Optical Wireless Network for Cellular Backhauling
}

\author{
Yuan Li, Student Member, IEEE, Nikolaos Pappas, Member, IEEE, Vangelis Angelakis, Member, IEEE, \\ Michał Pióro, Senior Member, IEEE, and Di Yuan, Member, IEEE
}

\begin{abstract}
With the densification of nodes in cellular networks, free space optic (FSO) connections are becoming an appealing low cost and high rate alternative to copper and fiber backhaul solutions for wireless communication systems. To ensure a reliable cellular backhaul, provisions for redundant, disjoint paths between the nodes must be made in the design phase. This paper aims at finding a cost-effective solution to upgrade the cellular backhaul with pre-deployed optical fibers using FSO links and mirror components. Since the quality of the FSO links depends on several factors, such as transmission distance, power, and weather conditions, we adopt an elaborate formulation to calculate link reliability. We present a novel integer linear programming model to approach optimal FSO backhaul design, guaranteeing $K$-disjoint paths connecting each node pair. Next, we derive a column generation method to a path-oriented mathematical formulation. Applying the method in a sequential manner enables high computational scalability. We use realistic scenarios to demonstrate our approaches efficiently, provide optimal or near-optimal solutions, and thereby allow for accurately dealing with the trade-off between cost and reliability.
\end{abstract}

Index Terms-FSO, mirrors, cellular backhauling, mixed integer programming, column generation, $K$-connectivity.

\section{INTRODUCTION}

A cellular backhaul comprises the connections between base stations and the core network components (e.g., radio network controller and base station controller). With high-speed data services and network densification using small cells, the need of upgrading the backhaul and increasing its capacity is rapidly growing [1]. At present, three transport media are primarily used for backhaul solutions: copper (about $90 \%$ ), microwave radio links (about 6\%), and optical fibers

Y. Li was with the Department of Electrical and Information Technology, Lund University, SE-22100 Lund, Sweden. He is now with the School of Computer, National University of Defense Technology, 410073 Changsha, China (email: yuan.li@nudt.edu.cn).

M. Pióro is with the Institute of Telecommunications, Warsaw University of Technology, Nowowiejska 15/19, 00-665 Warsaw, Poland (email: mpp@tele.pw.edu.pl), and also with the Department of Electrical and Information Technology, Lund University, SE-22100 Lund, Sweden.

N. Pappas, V. Angelakis and D. Yuan are with the Department of Science and Technology, Linköping University, SE-60174 Norrköping, Sweden. (emails: \{nikolaos.pappas, vangelis.angelakis, di.yuan\}@liu.se). D. Yuan is also visiting professor at the Institute for Systems Research, University of Maryland, College Park, MD 20742, USA.

The authors would like to acknowledge networking support by the COST Action IC1101 OPTICWISE (Optical Wireless Communications - An Emerging Technology). The work of M. Pióro was supported by National Science Center (Poland) under grant 2011/01/B/ST7/02967. Yuan Li was also supported by NFSC 61303264 and NFSC 61202482. Nikolaos Pappas was supported by the EU Marie Curie Actions project SOrBet (FP7-PEOPLE2013-IAPP: 612361). This work has been supported in part by the EU Marie Curie Actions project MESH-WISE (FP7-PEOPLE-2012-IAPP: 324515). (about 4\%) [2]. Leased copper lines are becoming an infeasible options for meeting future backhaul demands, as the data rate is low while the price increases linearly with capacity. On the other hand, optical fiber can support very high data rates but needs substantial initial investment. Conventional radio-frequency (RF) technologies have been widely studied for backhauling, and the RF mesh networking paradigm has attracted significant attention for backhaul topology. However, RF links have rather limited data rates, and are prone to interference and security problems. As frequencies go up with millimeter waves, RF transmissions are also hampered by distance and even weather conditions [3]. What is more, the licensed part of the spectrum comes at additional costs [4].

An FSO link uses the free space between a pair of laserphotodetector transceivers to transport data. The FSO beam has a wavelength in the micrometer range, yielding advantages in terms of free license, interference immunity, and high capacity, among others. Such FSO wireless transceivers are already commercially available [5], and can be deployed to establish optical links to support several gigabit per second over a distance of a few kilometers with fast deployment. FSO links are considerably more cost-efficient than optical fibers. As they can operate with similar wavelength, FSO and optical fibers can be easily combined together in a network [6]. With the advantages it brings, the FSO technology is a good complementary option for traditional radio-based wireless technologies. However, there are also inherent difficulties for the deployment of an FSO-based system. FSO links can be set up only when both end nodes are in the line of sight, and the link availability is sensitive to weather conditions, such as fog and precipitation [7]. Thus the aspect of reliability is of high significant in deploying an FSO-based network. In addition, from a network deployment standpoint, setting up FSO communication systems is challenging as there is a lack of modeling and computational approaches for topology optimization, which forms the scopes of the current paper.

In this paper, we address the network optimization problem of upgrading a fiber optical backhaul with FSO technology. Since optical fibers are insensitive to weather conditions, any pair of nodes connected by an optical fiber is treated as reliable. For FSO links, on the other hand, reliability is explicitly considered in our system model. A specific aspect in the network design problem we consider is the possible use of mirrors. When two FSO nodes are not in the line of sight, mirrors installed at a third node may potentially provide connectivity. An extension is to connect two FSO nodes with a sequence of mirrors. However, as mirrors do not provide 
any amplification, the total distance of such a mirror path is subject to a limit. The network design optimization problem consists in determining the locations of setting up FSO links and mirror links to provide connectivity, possibly via multiple hops, between all FSO nodes with traffic demand. A specific design concern is to guarantee a desired degree of resilience to cope. That is, the FSO nodes remain connected even if some of the wireless optical links become unavailable. This, so called network survivability, is formulated using $K$-connectivity, i.e., there are (at least) $K$ link-disjoint paths connecting each pair of FSO nodes, where $K$ is a parameter. A network topology example with 2-connectivity is shown in Fig. 1.

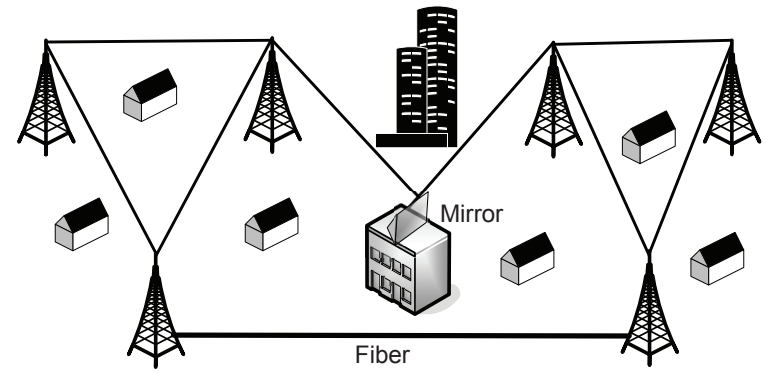

Fig. 1: A backhaul network deployment scenario with FSO transceivers, mirrors and fibers.

The performance objectives are deployment cost and network reliability. The former ranges from the cost of FSO transceivers, mirrors, to that of leasing building roofs for installing mirrors, whereas the latter is dependent on link lengths and the number of links. Considering these aspects along with $K$-connectivity lead to a comprehensive system model. We present following contributions to the FSO network optimization problem. First, we derive a novel integer linear programming approach that deals with the cost, reliability, and $K$-connectivity aspects using FSO and mirror links. The approach guarantees global optimality for up to medium-size planning scenarios. Next, for better scalability when dealing with large-size scenarios, we develop an alternative, pathoriented optimization formulation. This formulation allows for problem decomposition, by making use of a column generation method that finds candidate paths for each FSO node pair in an incremental manner. Our second solution approach consists in applying the column generation method sequentially to the FSO node pairs, to enable obtaining near-optimal solutions time-efficiently. We report computational results for realistic planning scenarios of FSO backhaul design, to demonstrate the viability of the optimization framework as well as to shed light on the trade-off between cost and reliability.

The FSO system considered in this paper operates in the wavelength of $1550 \mathrm{~nm}$, which is also widely used in the optical telecommunications [7]. To connect nodes that are not in line of sight, mirrors are used. Although there are other ways to provide non-line-of-sight propagation, for example, the ultraviolet-based transmission proposed in [8], we do not consider its deployment though. The ultraviolet communication operates in wavelength $4-400 \mathrm{~nm}$, which requires higher eye safety criteria. For this reason, more power can be transmitted by the laser with the wavelength of $1550 \mathrm{~nm}$ without exceeding the eye safety threshold, which is beneficial to overcome attenuation caused by fog. Moreover, the laser with shorter wavelength suffers from larger atmospheric attenuation and shorter transmission range. Nevertheless, due to line-of-sight transmissions, our system has a requirement on keeping alignment between any pair of nodes. Pointing errors can be introduced by the movement of transceivers and mirrors. Therefore, an automatic beam pointing, acquisition and tracking (PAT) mechanism with associated protocol and software are required at each transceiver and each mirror. Such systems have been proposed and studied in the literature, see for example [9], [10]. In our model these errors do not affect link reliability, only link outage probability which in turn affects maximum link distance.

The motivation for our work is twofold. First, free space optics (FSO)-based networking is an attractive alternative for next generation cellular networking [11]. Backhaul solutions based on FSO, carry the benefits we discussed earlier in the FSO link presentation. Second, the network design problem we consider is $N P$-hard because the special case of this problem without mirror nodes is an instance of the problem of finding the minimal $K$-edge-connected graph which is known to be $N P$-hard [12]. To the best of our knowledge, there are no trivial methods to find even a feasible solution for our considered problem. Thus, the models and method proposed in this work are the first for this problem, and their benefits in reducing the complexity of solving the problem and saving deployment cost will be analyzed in the numerical study.

The remainder of the section is organized as follows. Related works are reviewed in Section II. Section III introduces the FSO channel model and basic notations. The exact integer programming model is presented in Section IV, and then in Section $\mathrm{V}$, the sequential computation approach is elaborated. The numerical study illustrating optimal solutions and comparing algorithms is conducted in Section VI. Finally, Section VII concludes the paper.

\section{RELATED WORK}

In the survivable network design there is a significant body of works. Various mathematical programming models have been proposed for designing $K$-connected graphs, see for example [13] and references therein. Papers [14], [15] study the problem of $K$-connected network design with node degree constraint or limiting the number of hops. An algorithm for survivable network design with reliable links, which can be shared by link-disjoint paths is proposed in [16]. In our paper, we consider the design of a $K$-connected graph with both reliable links (optical fibers) and non-reliable links, i.e., FSO links and mirror links. Furthermore, we constrain the total length of consecutive mirror links, which may only be part of a path connecting a pair of FSO transceivers. This constraint differentiates our work from the traditional problem limiting the number of hops per path.

The concept of $K$-connected graph has been applied in the research on wireless sensor networks before. For example, paper [17] considers designing a $K$-connected network to serve as the backbone for wireless sensor networks by means 
of a $m$-domination constraint. The dominating set is a subset of nodes such that each node outside this set has at least one neighbor in the set, and the $m$-domination constraint requires that each node outside the set has at least $m$ neighbors in the set. Furthermore, some distributed and approximate algorithms are proposed in [18]. Other problems, such as energy saving, target coverage and area coverage, are studied by means of fault-tolerant network design, i.e, guaranteeing $K$ connectivity in [19]. A recent survey paper [20] investigates algorithms for designing $K$-connected sensor networks by placing redundant sensor nodes. However, up to now no work has been done for studying $K$-connectivity imposing a limitation on the consecutive use of relay nodes (mirrors) - this is a theoretical contribution of this work and a difficult part in network modeling.

With FSO being an affordable alternative to optical fibers for high data-rate communication, there is a vast amount of research focused on FSO networking. The majority of works studies the factors (weather, alignment, turbulence, etc.) that affect the link performance and also investigates different techniques (modulation and coding schemes, electronics design, etc.) to improve the FSO link quality (see for example [21] and the references therein). On the other hand, work that focuses on network optimization aspects on FSO communications are rather limited. Recently though the problem of network topology design has attracted attention. In [22]-[24] simple topology design models have been studied for the FSO networks. The work [22] develops heuristics to design 2-degree topologies (2 transceivers per node) and 3degree topologies (3 transceivers per node) with a minimum number of links. Paper [23] adopts a combination of multiple objectives, i.e., minimizing the cost in the physical layer and the congestion in the logical layer to generate a 2-degree topology heuristically. The paper [24] presents an integer programming model to maximize the network throughput by installing as many as possible FSO links when the number of optical transceivers per node is limited. Similarly [25] proposes algorithms for integrated topology control and singlepath or multi-path routing. In our work, we do not restrict the number of FSO transceivers in each node since in practice, as FSO transceivers can be collocated (being mounted at different heights) in the same node.

Integer programming models for designing topologies based on FSO have also been proposed. In [26] the backbone of wireless mesh networks with FSO links is designed by maximizing the so called algebraic connectivity. However, the algebraic connectivity cannot capture the resilience of connections for each pair of nodes. The same objective is also studied in paper [27]. The [28] presents a model that performs load balancing and provides link-disjoint paths for each pair of nodes. However, the proposed simple algorithm for finding link-disjoint paths does not take into account that finding shortest path one-by-one may not produce feasible link-disjoint paths (see appendix C.4.1 in [29]). Joint topology design and load balancing in FSO networks is addressed in [30] where the reformulation linearization technique (RLT) is applied to obtain linear programming (LP) relaxations of the original complex problem, and then incorporate the LP relaxations into a branch-and-bound framework.

\section{SYSTEM MODEL}

\section{A. Link Model}

FSO is an optical wireless communication technology that uses lasers propagating in the free space to transmit data between two FSO transceivers. In this paper we assume intensity modulation and direct detection is adopted [31]. During propagation, the laser beam is attenuated due to photon absorption and scattering, fog, wind, temperature, pointing errors, etc. [7]. The path loss for FSO transmissions can be computed by the Friis transmission equation in (1) [32], where $P_{T}, n_{T}, G_{T}$ are the optical power, efficiency, and telescope gain of the transmitter and $P_{R}, n_{R}, G_{R}$ of the receiver, respectively. $h$ is the channel state, computed by $h=h_{l} h_{a} h_{p}$ where $h_{l}, h_{a}, h_{p}$ represent the atmospheric attenuation, the atmospheric turbulence and pointing errors, respectively. Our model, although independent of the exact values of these errors, considers them in the outage probability within (1) for accounting maximum link length. The analytical model of our pointing errors comes from equations (9)-(11) of [33].

$$
P_{R}=P_{T} n_{T} n_{R} G_{T} G_{R}\left(\frac{\lambda}{4 \pi l}\right)^{2} h=A h .
$$

Note that $A=P_{T} n_{T} n_{R} G_{T} G_{R}\left(\frac{\lambda}{4 \pi l}\right)^{2}, \lambda$ is the wavelength and $l$ is the transmission distance. If a pair of FSO transceivers is connected by a line-of-sight link, $l$ refers to the length of this link. If they are connected though several consecutive mirrors, $l$ is the length of such a mirror path.

The probability distribution of channel state $h$, i.e, $f_{h}(h)$, has been modelled in [33], and based on that we can compute the outage probability by (2), where $h_{0}=\bar{P} / A$, $\sigma_{X}^{2} \approx 0.30545\left(\frac{2 \pi}{\lambda}\right)^{7 / 6} C_{n}^{2} l^{11 / 6}, \phi=\ln \left(h_{0} / A_{0} h_{l}\right)+\mu, A_{0}=$ $[\operatorname{erf}(v)]^{2}, h_{l}=\exp (-\sigma l), \mu=2 \sigma_{X}^{2}\left(1+2 \gamma^{2}\right), \gamma=\omega_{z e q} /\left(2 \sigma_{s}\right)$, $\omega_{z e q}^{2}=\omega_{z}^{2} \sqrt{\pi} \operatorname{erf}(v) /\left(2 v \exp \left(-v^{2}\right)\right), v=\sqrt{\pi} a /\left(\sqrt{2} \omega_{z}\right)$, $\omega_{z} \approx\left[1+\zeta\left(\frac{\lambda l}{\pi \omega_{0}^{2}}\right)\right]^{0.5}$. The explanations of all parameters used for a link budget analysis are listed in Table I, which are derived from papers [33]-[35].

$$
\begin{aligned}
& P_{\text {out }}=\operatorname{Pr}\left(P_{R} \leq \bar{P}\right)=\operatorname{Pr}(h \leq \bar{P} / A)=\int_{0}^{h_{0}} f_{h}(h) d h= \\
& \frac{1}{2}\left[e^{\gamma^{2} \phi-2 \sigma_{X}^{2} \gamma^{4}} \operatorname{erfc}\left(\frac{\phi}{\sqrt{8} \sigma_{X}}\right)+\operatorname{erfc}\left(\frac{4 \sigma_{X}^{2} \gamma^{2}-\phi}{\sqrt{8} \sigma_{X}}\right)\right] .
\end{aligned}
$$

We make the link budget analysis in terms of transmission distance versus the outage probability and the jitter standard deviation $\left(\sigma_{s}\right)$, which is shown in Fig. 2. As we can see, the outage probability increases with the increasing of $\sigma_{s}$. Taking $\sigma_{s}=12 \mathrm{~cm}$, the outage probability is below $10^{-6}$ if the transmission distance is smaller than $1400 \mathrm{~m}$. We will use it as the maximum transmission distance in the numerical study, i.e., a direct line-of-sight link or a path consisting of mirrors connecting a pair of FSO transceivers has a maximum length of $1400 \mathrm{~m}$.

Note that we assume that perfect automatic tracking systems can be used for FSO transceivers and mirrors, thus such maximum link length in fact corresponds to a much lower 
TABLE I: Parameters used for link budget analysis.

\begin{tabular}{lcclcc}
\hline Optical transmission power & $P_{T}$ & $10 \mathrm{~mW}$ & Receiver diameter & $2 a$ & $0.2 \mathrm{~m}$ \\
Minimum required receiving power & $\bar{P}$ & $10^{-7} \mathrm{~W}$ & Laser wavelength & $\lambda$ & $1550 \mathrm{~nm}$ \\
Transmitter/Receiver efficiency & $n_{T} / n_{R}$ & $0.9 / 0.9$ & Jitter standard deviation & $\sigma_{s} \cong 12 \mathrm{~cm}$ \\
Laser transmitter/receiver telescope gain & $G_{T} / G_{R}$ & $72 / 112 \mathrm{~dB}$ & Beam radius at $0 \mathrm{~m}$ & $w_{0}$ & $\cong 70 \mathrm{~cm}$ \\
The source coherence parameter & $\zeta$ & 1 & Responsively & $R$ & 0.5 \\
Refractive-index structure & $C_{n}^{2}$ & $10^{-15} \mathrm{~m}^{-\frac{2}{3}}$ & Visibility & $10 \mathrm{~km}$ \\
Attenuation coefficient & $\sigma$ & 0.1 & & \\
\hline
\end{tabular}

outage probability. The cost of such system can be considered to be reflected within the coefficient of FSO transceivers in our model.

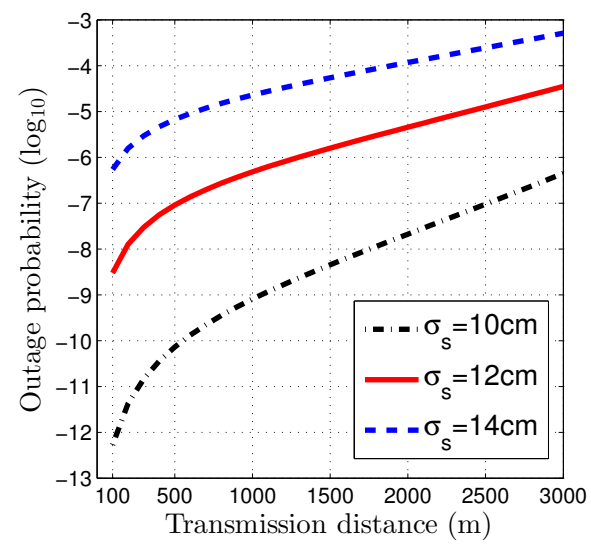

Fig. 2: Outage probability versus the distance for three different values of the jitter standard deviation.

In this paper we introduce the concept of link reliability to consider the factor of atmospheric turbulence in the network design. Pointing errors are not taken into account due to the assumption that automatic tracking systems are used. The probability density function of the irradiance $I$ is characterized by the log-normal model, which is expressed in (3) [36].

$$
p(I)=\frac{1}{2 \sqrt{2 \pi} I \sigma_{X}} \exp \left(-\frac{\left(\ln I-\ln I_{0}\right)^{2}}{8 \sigma_{X}^{2}}\right) .
$$

The reliability of a link is defined as the cumulative probability of the irradiance above a threshold of the received signal intensity $I_{t h}$ and can be computed by equation (4), where $I_{0}$ is the average irradiance when there is no turbulence [26].

$$
\Gamma(l)=\int_{I_{t h}}^{\infty} p(I) d I=\frac{1}{2}-\frac{1}{2} \operatorname{erf}\left(\frac{\ln \left(I_{t h} / I_{0}\right)}{2 \sigma_{X} \sqrt{2}}\right) .
$$

\section{B. Network Model}

For the problem considered in this paper we assume that an initial (undirected) network graph is given, including a set of nodes $\mathcal{V}$ and a set of potential links $\mathcal{E}$. The set of nodes consists of the set of the base stations for deploying FSO transceivers (FSO nodes, $\mathcal{V}^{F}$ ) and the buildings (mirror nodes, $\mathcal{V}^{M}$ ) on which mirrors can be deployed, i.e., $\mathcal{V}=\mathcal{V}^{F} \cup \mathcal{V}^{M}$ where $\mathcal{V}^{F}, \mathcal{V}^{M}$ are disjoint. There are three types of links: fiber links, FSO links, and mirror links. The set of all fiber links is denoted by $\mathcal{E}^{O}$, the set of all potential FSO links - by $\mathcal{E}^{F}$, and the set of all potential mirror links - by $\mathcal{E}^{M}$. The set of links is thus defined as $\mathcal{E}=\mathcal{E}^{O} \cup \mathcal{E}^{F} \cup \mathcal{E}^{M}$ where the sets $\mathcal{E}^{O}, \mathcal{E}^{F}$ and $\mathcal{E}^{M}$ are disjoint. Links are undirected and the end nodes of link $e \in \mathcal{E}$ are denoted by $p_{e}, q_{e} \in \mathcal{V}$.

The fiber links constituting set $\mathcal{E}^{O}$ are just links between FSO nodes established over the fibers and are assumed to be already deployed in the considered backhaul network. Each FSO link $e \in \mathcal{E}^{F}$ connects two FSO nodes $p_{e}$ and $q_{e}$, which can also possibly be connected by several parallel mirror paths. Each such a mirror path is composed of a sequence (possibly empty) of mirror links and is equipped with two FSO transceivers placed in its end nodes $\left(p_{e}\right.$ and $\left.q_{e}\right)$, and the mirrors in the relay (mirror) nodes. When the mirror path is empty then two FSO transceivers of link $e$ are connected by a direct laser beam (the end nodes must be in the line of sight). In a mirror link $e \in \mathcal{E}^{M}$ at least one end node is a mirror node and the other is either another mirror node or an FSO node. The FSO links with the end nodes not in the line of sight are represented by the subset $\mathcal{E}^{N} \subseteq \mathcal{E}^{F}$.

The sets of bi-directed arcs corresponding to the sets of undirected fiber links, FSO and mirror links are denoted by $\mathcal{A}^{O}, \mathcal{A}^{F}, \mathcal{A}^{M}$, respectively. Each undirected link $e \in \mathcal{E}$ corresponds two oppositely directed arcs $a^{\prime}, a^{\prime \prime} \in \mathcal{A}$. We define a mapping $f: \mathcal{A} \rightarrow \mathcal{E}$ such that $f\left(a^{\prime}\right)=e, f\left(a^{\prime \prime}\right)=e$ and $f^{-1}(e)=\left\{a^{\prime}, a^{\prime \prime}\right\}$. We use $\delta_{a v}^{+}$to represent whether arc $a$ is outgoing from node $v, \delta_{a v}^{+}=1$, or not, $\delta_{a v}^{+}=0$. $\delta_{a v}^{-}$ indicates whether $\operatorname{arc} a$ is incoming to node $v, \delta_{a v}^{-}=1$, or not, $\delta_{a v}^{-}=0$. Two paths with the same end nodes are called link-disjoint when they have no FSO link nor mirror link in common, but may contain the common fiber links. We aim at constructing a subgraph that assures $K$ link-disjoint paths for each pair of FSO nodes, meaning that the subgraph is $K$ connected in this sense. We formulate the problem by utilizing a multi-commodity flow model. Each pair of FSO nodes is treated as a commodity; the set of all such commodities is denoted by $\mathcal{D}$. The source and the destination of a commodity $d$ are represented by $s_{d} \in \mathcal{E}^{F}$ and $t_{d} \in \mathcal{E}^{F}$, respectively.

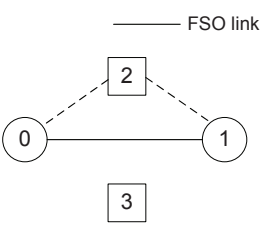

(a)

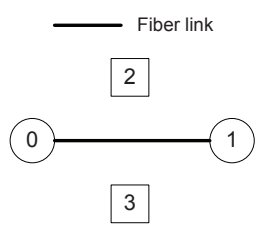

(b)

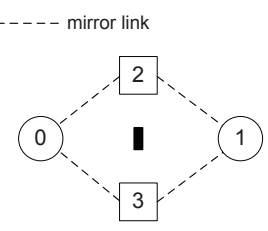

(c)
Fig. 3: An example of 2-connected pairs.

In the considered model, FSO transceivers, fibers and mirrors play different roles in the construction of the $K$-connected graph. A direct FSO link or a mirror link can be established 
only when there is the line of sight between their end-nodes. The fiber links are assumed to already exist and we just use them for designing the paths. For a given commodity, the paths sharing a fiber link are treated as link-disjoint, since fibers are much more reliable than FSO and mirror links. The mirror nodes are used to connect FSO nodes which are not in the line of sight, or to create parallel paths to assure path diversity. Note that not all mirror nodes will in general be used in the optimal solution. When a mirror path traverses a mirror node, it is assigned its own mirror at that node. Fig. 3 (where circles, squares and black rectangles represent FSO nodes, mirror nodes and obstacles, respectively) illustrates three cases of link-disjoint paths between nodes 0 and 1: (a) one path consists of mirror links and the other path consists of an FSO link; (b) an optical fiber can be treated as a pair of link-disjoint paths; (c) two link-disjoint paths consists of sole mirror links due to an obstacle between node 0 and node 1 .

\section{PROBLEM DESCRIPTION AND FORMULATION}

In this section we formulate the basic problem of this paper. Roughly speaking, the problem consists in constructing a $K$-connected subgraph while optimizing the number of the required FSO transceivers and mirrors for a given (potential) network graph with already installed fiber links. In the formulation we impose an additional requirement that the length of the mirror path is limited by a maximum transmission distance $L$. The problem is formulated as a two-layer network model where the upper layer consists of the FSO links, fiber links and FSO nodes. The lower layer consists of the mirror links and all nodes. This two-layer model is illustrated by an example in Fig. 4.

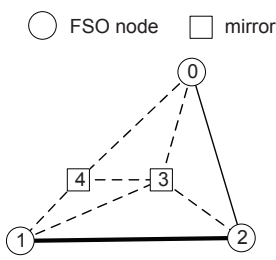

(a) a graph

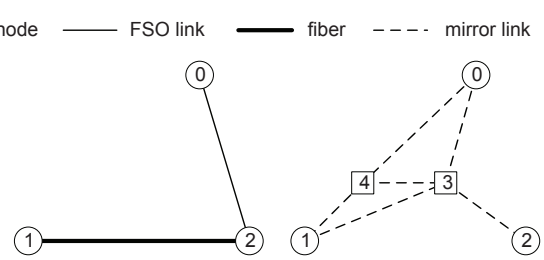

(b) the upper and lower layer
Fig. 4: An illustration of two layers for a graph.

Before proceeding to the problem formulation, we note that the strength of an optical signal is fixed when the signal is sent from an FSO transceiver (it is transmitted with a fixed power) and this strength is not increased when it is reflected by a mirror. This is illustrated in Fig. 5(b). Path $(0,3,1)$ is not feasible since the total length of link $\{0,3\}$ and link $\{3,1\}$ is excessive. However, path $(0,3,2,3,1)$ is feasible since the signal will arrive firstly at the FSO node 2 and then will be re-transmitted with the maximum strength. In fact, this path consists of two feasible mirror paths $(0,3,2)$ and $(2,3,1)$. A mirror can serve different commodities, for example a mirror in node 4 of Fig. 5(a) serves commodities $(0,3)$ and $(1,3)$. Also, a signal incoming to a mirror cannot be split and forwarded to two different directions. Thus two mirrors are deployed at node 3 in Fig. 5(b) to establish two parallel beams. Note that case (a) requires 10 FSO transceivers and 2 mirrors while case (b) requires 8 FSO transceivers and 2 mirrors.

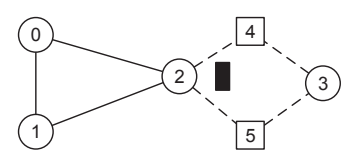

(a) case 1

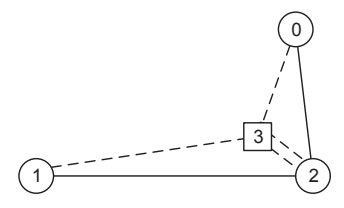

(b) case 2
Fig. 5: Optimal 2-connected topologies with the usage of mirrors.

In the formulation each commodity is divided to $K$ flows and the corresponding $K$ link-disjoint paths so that each flow is transmitted over one path. To set up the formulation, we define the following variables.

$x_{a}^{k d}$ binary variable indicating whether the $k$-th flow of the commodity $d \in \mathcal{D}$ passes through the FSO arc $a \in \mathcal{A}^{F}$.

$w_{e}$ integer variable indicating the maximum number of flows of a commodity between the end nodes of the FSO link $e \in \mathcal{E}^{F}$.

$u_{e}$ binary variable indicating whether the direct beam connection is established for link $e \in \mathcal{E}, u_{e}=1$ (then the end nodes of $e$ must be in the line of sight), or not, $u_{e}=0$.

$U_{e}$ binary variable indicating whether the link $e \in \mathcal{E}$ carries traffic, $U_{e}=1$, or not, $U_{e}=0$.

$R_{e}^{k d}$ binary variable indicating whether the link $e \in \mathcal{E}$ is used (or not) for the $k$-th flow of commodity $d, R_{e}^{k d}=1$ $\left(R_{e}^{k d}=0\right)$.

$y_{e} \quad$ integer variable expressing the number of mirrors used to connect the end nodes of an FSO link $e \in \mathcal{E}^{F}$.

$r_{e}^{k d}$ binary variable indicating whether a mirror path corresponding to the FSO link $e \in \mathcal{E}^{F}$, for the $k$-th flow of commodity $d$, should be established, $r_{e}^{k d}=1$, or not, $r_{e}^{k d}=0$.

$z_{m e}^{k d}$ binary variable indicating whether the mirror arc $m \in$ $\mathcal{A}^{M}$ is used by a mirror path realizing the FSO link $e \in \mathcal{E}^{F}$, for the $k$-th flow of the commodity $d, z_{m e}^{k d}=1$, or not $z_{m e}^{k d}=0$.

$Z_{e^{\prime} e}^{k d}$ binary variable indicating whether the mirror link $e^{\prime} \in$ $\mathcal{E}^{M}$ is used by a mirror path realizing FSO link $e \in \mathcal{E}^{F}$, for the $k$-th flow of the commodity $d, Z_{e^{\prime} e}^{k d}=1$, or not, $Z_{e^{\prime} e}^{k d}=0$.

$X_{v}$ binary variable indicating whether the mirror node $v \in$ $\mathcal{V}^{M}$ is used for deploying mirrors, $X_{v}=1$, or not $X_{v}=$ 0 .

The objective function is given by

$$
\begin{aligned}
\text { Minimize } & c_{1} \sum_{v \in \mathcal{V}^{F}} \sum_{e \in \mathcal{E}^{F}} \Delta_{e v} w_{e}+c_{2} \sum_{v \in \mathcal{V}^{M}} X_{v}+ \\
& c_{3} \sum_{e \in \mathcal{E}^{F}} y_{e}-c_{4} \sum_{e \in \mathcal{E} \backslash \mathcal{E}^{O}} \kappa_{e} u_{e} .
\end{aligned}
$$

This is a multi-objective function and the positive coefficients $c_{1}, c_{2}, c_{3}, c_{4}$ are the weights of the four terms. In (5), the first term is the number of the FSO transceivers, where $w_{e}$ denotes the maximum number of flows of a commodity connecting the end nodes of FSO link $e$. These flows should use link-disjoint paths, i.e., each flow is allocated either an FSO link or a mirror 
path. Since each flow starts from an FSO transceiver and ends at an FSO transceiver, the number of FSO transceivers needed for each flow is $\sum_{v \in \mathcal{V}^{F}} \Delta_{e v} w_{e}$. Then the total number of FSO transceivers, that will be used in the optimal solution, is $\sum_{v \in \mathcal{V}^{F}} \sum_{e \in \mathcal{E}^{F}} \Delta_{e v} w_{e}$. The second term denotes the number of used mirror nodes. The third term denotes the number of mirrors which is the sum of all mirrors used in the mirror paths for all FSO links, i.e., $\sum_{e \in \mathcal{E}^{F}} y_{e}$. The first three terms are related to the deployment cost, and $c_{1} \gg c_{2} \gg c_{3}$ since the cost of an FSO transceiver is higher than the leasing cost of building roof, followed by the cost of mirrors. The fourth term is the network reliability, which is the sum of the reliability over all links. The reliability of link $e$ is denoted by $\kappa_{e}\left(0 \leq \kappa_{e} \leq 1\right)$, which is equal to $\Gamma\left(l_{e}\right)$ (computed by equation (4)) where $l_{e}$ is the length of link $e$. To maximize the network reliability, a minus sign is placed before the term. Our objective is to balance the deployment cost and the system reliability.

To create a $K$-connected pair of FSO nodes, we transmit $K$ flows, with unitary volume for each path, from the source to the destination. This is formulated in (6), which is the flow conservation constraint.

First-layer multicommodity flow constraints:

$$
\begin{gathered}
\sum_{a \in \mathcal{A}^{F} \cup \mathcal{A}^{O}}\left(\delta_{a v}^{+} x_{a}^{k d}-\delta_{a v}^{-} x_{a}^{k d}\right)=\left\{\begin{array}{l}
1, v=s_{d} \\
-1, v=t_{d} \\
0, \text { otherwise. }
\end{array}\right. \\
k \in[1, K], v \in \mathcal{V}^{F}, d \in \mathcal{D}
\end{gathered}
$$

The $K$ flows of a commodity have to use $K$ link-disjoint paths. The link-disjoint constraints on FSO links are expressed in (7). Link-disjoint constraint on FSO links and fiber links:

$$
\begin{array}{ll}
x_{a}^{k d} \leq R_{f(a)}^{k d}+r_{f(a)}^{k d}, & \\
\quad a \in \mathcal{A}^{F}, d \in \mathcal{D}, k \in[1, K] & \\
\sum_{k \in[1, K]} R_{e}^{k d} \leq u_{e}, & e \in \mathcal{E}^{F}, d \in \mathcal{D} \\
\sum_{k \in[1, K]} \sum_{d \in \mathcal{D}} \sum_{a \in f^{-1}(e)} x_{a}^{k d} \geq u_{e} & e \in \mathcal{E}^{F} \\
\sum_{k \in[1, K]} \sum_{d \in \mathcal{D}} R_{e}^{k d} \geq u_{e} & e \in \mathcal{E}^{F} \\
u_{e}=0, & e \in \mathcal{E}^{N} .
\end{array}
$$

If an FSO arc $a$ carries the $k$-th flow of commodity $d$, either an FSO link, indicated by $R_{f(a)}^{k d}$, is used or a mirror path, indicated by $r_{f(a)}^{k d}$, is established (7a). At most one flow realizing a given commodity can pass through a given FSO link-this is assured by constraint (7b). (Note that they can share a fiber link.) Further, constraints (7c) and (7d) make sure that link $e$ is not established when it does not carry any flows using the direct beam (this is important from the viewpoint of last term in the objective function). A link should also not be established if there is no line of sight, which is assured by (7e).

Constraint (8) expresses the maximum number of flows belonging to a commodity between end nodes of FSO link $e$ over all commodities.

Counting the maximum number of flows of a commodity between end nodes of an FSO link:

$$
\sum_{a \in f^{-1}(e)} \sum_{k \in[1, K]} x_{a}^{k d} \leq w_{e}, \quad e \in \mathcal{E}^{F}, d \in \mathcal{D} .
$$

In the second layer of the model, if $r_{e}^{k d}=1$, then a mirror path that connects the end nodes of an FSO link $e$ for commodity $d$ must be found. This is addressed by the constraint (9a). Constraint (9b) assures that a mirror path cannot pass through any FSO nodes, except the origin and the destination. Constraint (9c) expresses that when a mirror arc $m$ is used in a mirror path for FSO link $e$, which carries the $k$-th flow of commodity $d$, then the corresponding link $f(m)$ is used for the $k$-th flow of commodity $d$.

Second-layer multicommodity flow constraints:

$$
\begin{aligned}
& \sum_{m \in \mathcal{A}^{M}}\left(\delta_{m v}^{+} z_{m e}^{k d}-\delta_{m v}^{-} z_{m e}^{k d}\right)=\left\{\begin{array}{l}
r_{e}^{k d}, v=p_{e} \\
-r_{e}^{k d}, v=q_{e} \\
0, v \in \mathcal{V}^{M}
\end{array}\right. \\
& k \in[1, K], e \in \mathcal{E}^{F}, d \in \mathcal{D} \\
& \sum_{m \in \mathcal{A}^{M}}\left(\delta_{m v}^{+}+\delta_{m v}^{-}\right) z_{m e}^{k d}=0, \\
& k \in[1, K], \quad v \in \mathcal{V}^{F} \backslash\left\{p_{e}, q_{e}\right\}, e \in \mathcal{E}^{F}, d \in \mathcal{D} \\
& z_{m e}^{k d} \leq Z_{f(m)}^{k d}, \quad k \in[1, K], m \in \mathcal{A}^{M}, e \in \mathcal{E}^{F}, d \in \mathcal{D} .
\end{aligned}
$$

At most one of the flows realizing a given commodity can use a given mirror link - this is assured by (10a) since $u_{e}$ is binary. This constraint assures also that a mirror link $e$ is established when there are some flows on it; if there are no flows using mirror link $e$ then it is not which is assured by (10b).

Disjoint mirror links constraints:

$$
\begin{aligned}
& \sum_{k=1}^{K} Z_{e}^{k d} \leq u_{e}, d \in \mathcal{D}, e \in \mathcal{E}^{M} \\
& \sum_{d \in \mathcal{D}} \sum_{k=1}^{K} \sum_{e^{\prime} \in \mathcal{E}} \sum_{m \in f^{-1}(e)} z_{m e^{\prime}}^{k d} \geq u_{e}, e \in \mathcal{E}^{M} .
\end{aligned}
$$

The inequality (11) expresses the number of mirrors used to connect end nodes of FSO link $e$. In the left hand side, the term $\sum_{m \in \mathcal{A}^{M}} z_{m e}^{k d}-r_{e}^{k d}$ denotes the number of mirrors used in a mirror path for FSO link $e$ deployed by the $k$-th flow of a commodity $d$. Then, for a commodity $d$, the number of mirrors needed in order to connect the end nodes of FSO link $e$ is the summation over the number of mirrors for all the flows. Then, that number is the maximal number of mirrors needed for all the commodities, as described in the following remark.

\section{Counting the number of mirrors:}

$$
\sum_{k=1}^{K}\left(\sum_{m \in \mathcal{A}^{M}} z_{m e}^{k d}-r_{e}^{k d}\right) \leq y_{e}, e \in \mathcal{E}^{F}, d \in \mathcal{D} .
$$

Remark: the number of deployed mirrors between the end nodes of an FSO link is the maximum number of mirrors needed over all the commodities.

If a flow passes through the end nodes of FSO link $e$ and there is a need for a mirror path, i.e., $r_{e}^{k d}=1$, then the number of used mirrors is $\sum_{m \in \mathcal{A}^{M}} z_{m e}^{k d}-r_{e}^{k d}$. Note that if $r_{e}^{k d}=0$, then $z_{m e}^{k d}=0$ which is assured by (9a). The number of used mirrors for commodity $d$ on the FSO link $e$ is $\sum_{i=1}^{K}\left(\sum_{m \in \mathcal{A}^{M}} z_{m e}^{k d}-r_{e}^{k d}\right)$, since two or more flows cannot pass through the same mirror link. A mirror path for an FSO link can serve different commodities. Thus the number of used mirrors between the end nodes of an FSO link is the maximum number of mirrors needed over all the commodities.

The length of each mirror path should not be bigger than the maximum transmission distance, as the optical signal attenuates during its transmission along a mirror path. This is assured by inequality (12). 


\section{Limiting the length of a mirror path:}

$$
\sum_{m \in \mathcal{A}^{M}} l_{m} z_{m e}^{k d} \leq L, \quad k \in[1, K], e \in \mathcal{E}^{F}, d \in \mathcal{D} .
$$

If a mirror link is established, the corresponding mirror node should be leased; the previous is assured by (13).

\section{Mirror node constraint:}

$$
u_{e} \leq X_{v}, \quad v \in \mathcal{V}^{M}, e \in \mathcal{E}^{M}, \Delta_{e v}=1 .
$$

In summary, the mathematical formulation for cost-efficient and resilient backhaul network design utilizing FSO transceivers, mirrors and optical fibers (CRBND) is given below.

\section{CRBND Minimize (5) \\ s.t. $\quad(6),(7),(8),(9),(10),(11),(12),(13)$.}

\section{AN EFFICIENT SEQUENTIAL COMPUTATION APPROACH}

The time required to solve CRBND increases dramatically as the size of the network increases. Therefore, for large networks, we need to find an efficient and practical method. In this section, we propose a sequential computation approach, i.e., finding $K$ link-disjoint paths in a manner of commodityby-commodity.

The steps for this approach are given below.

Step 1. The commodities are sorted in the descending order based on the hops in the shortest path between the source and the destination. Thus, the commodity with the largest hops between the source and the destination is disposed firstly. The reason is that the commodity with the larger hops is inclined to uses more FSO transceivers and mirrors, which will be reused by subsequent commodities to save the total cost.

Step 2. For each commodity, we need to establish $K$ linkdisjoint paths. We develop a new non-compact formulation, called the link-path formulation. This formulation is based on the notation used for paths, which is shown in (14). We denote the set of available paths for the considered commodity as $\mathcal{P}$, define the constant $\Lambda_{e p}$ to represent whether link $e \in \mathcal{E}$ belongs to the path $p \in \mathcal{P}, \Lambda_{e p}=1$, or not $\Lambda_{e p}=0$, define the constant $\Theta_{a p}$ to represent whether arc $a \in \mathcal{A}$ belongs to the path $p \in \mathcal{P}, \Theta_{a p}=1$, or not $\Theta_{a p}=0$, and define $\delta_{a v}=\delta_{a v}^{+}+\delta_{a v}^{-}$.

The introduced variables are defined below.

$X_{p}$ binary variable, $X_{p}=1$ if path $p \in \mathcal{P}$ is used; $X_{p}=0$ otherwise.

$U_{e}$ binary variable, $U_{e}=1$ if link $e \in \mathcal{E}$ is established; $U_{e}=0$ otherwise.

$Y_{v}$ binary variable, $Y_{v}=1$ if mirror node $v \in \mathcal{V}^{M}$ is used; $Y_{v}=0$ otherwise.

The four terms in the objective function (14a) denote the number of FSO transceivers, the number of used mirror nodes, the number of mirrors and the network reliability. In this objective, the first term and the third term are different from their counterparts in (5), however the equivalence is proved in Proposition 1. The constraint (14b) assures that $K$ paths are selected and they are link-disjoint, i.e., two paths cannot share an FSO link or a mirror link, which is assured by constraint (14c). Constraint (14c) also assures that an FSO link or a mirror link is deployed if and only if there is a flow on it. Finally, constraint (14d) forces that a mirror node should be leased when there is a deployed link attached to it.

The link-path formulation is given as below.

$$
\begin{aligned}
\text { Minimize } & c_{1} \sum_{v \in \mathcal{V}^{F}} \sum_{a \in \mathcal{A} \backslash \mathcal{A}^{O}} \sum_{p \in \mathcal{P}} \delta_{a v} \Theta_{a p} X_{p} \\
& +c_{2} \sum_{v \in \mathcal{V}^{M}} Y_{v}-c_{4} \sum_{e \in \mathcal{E} \backslash \mathcal{E} O} \kappa_{e} U_{e} \\
& +\frac{c_{3}}{2} \sum_{a \in \mathcal{A}^{M}} \sum_{v \in \mathcal{V}^{M}} \sum_{p \in \mathcal{P}} \delta_{a v} \Theta_{a p} X_{p} \\
& \sum_{p \in \mathcal{P}} X_{p}=K \\
& \sum_{p \in \mathcal{P}} \Lambda_{e p} X_{p}=U_{e}, e \in \mathcal{E}^{F} \cup \mathcal{E}^{M} \\
& \Delta_{e v} U_{e} \leq Y_{v}, v \in \mathcal{V}^{M}, e \in \mathcal{E}^{M}
\end{aligned}
$$

Proposition 1. In the model (14), the number of FSO transceivers is $\sum_{v \in \mathcal{V}^{F}} \sum_{a \in \mathcal{A} \backslash \mathcal{A} O} \sum_{p \in \mathcal{P}} \delta_{a v} \Theta_{a p} X_{p}$ and the number of mirrors is $\frac{1}{2} \sum_{a \in \mathcal{A}^{M}} \sum_{v \in \mathcal{V}^{M}} \sum_{p \in \mathcal{P}} \delta_{a v} \Theta_{a p} X_{p}$.

Proof. A path in the optimal solution of (14) starts from an FSO node and terminates at another FSO node. The intermediate node can be a mirror node or an FSO node. Note that two oppositely directed arcs corresponding to a same link may appear in a path, e.g., path $(0,3,2,3,1)$ in Fig. 5(b). Thus, the total number of FSO transceivers for path $p$ can be counted in the following way: for each FSO node, count the number of attached arcs included in path $p$, and then sum up over all FSO nodes, which is expressed as $\sum_{v \in \mathcal{V}^{F}} \sum_{a \in \mathcal{A} \backslash \mathcal{A}^{O}} \delta_{a v} \Theta_{a p} X_{p}$. The number of mirrors in path $p$ is counted in a similar way: for each mirror node, count the number of attached mirror arcs included in path $p$, sum up over all mirror nodes and divide by 2 (a mirror corresponds two mirror links), which is expressed as $\frac{1}{2} \sum_{v \in \mathcal{V}^{M}} \sum_{a \in \mathcal{A}^{M}} \delta_{a v} \Theta_{a p} X_{p}$. The paths for one commodity are link-disjoint, meaning that each arc $a \in \mathcal{A}$ will not be used in different paths. Therefore, the total number of FSO transceivers (mirrors) can be obtained by summing up over all paths.

The optimization problem in (14) cannot be solved directly, since we cannot pre-define a set of paths where the optimal paths are included. A practical method is to solve its linear relaxation by path generation, i.e., iteratively generating paths until the optimum is achieved. Regarding the linear relaxation of (14), which is called master problem, let $\lambda, \pi_{e}, e \in \mathcal{E}$ denote the dual variables for constraints (14b) and (14c) respectively. Given an initial set of paths $\mathcal{P}^{*}$, we solve the master problem and obtain the optimal dual variables $\lambda^{*}, \pi_{e}^{*}, e \in \mathcal{E}$. In the given graph, if we can find a new path $p$, such that $\lambda>$ $c_{1} \sum_{v \in \mathcal{V}^{F}} \sum_{a \in \mathcal{A} \backslash \mathcal{A}^{O}} \delta_{a v} \Theta_{a p}+\frac{c_{3}}{2} \sum_{a \in \mathcal{A}^{M}} \sum_{v \in \mathcal{V}^{M}} \delta_{a v} \Theta_{a p}+$ $\sum_{e \in \mathcal{E}^{F} \cup \mathcal{E}^{M}} \Lambda_{e p} \pi_{e}$. Then, this path is added to the current set of paths and the master problem is solved again to obtain new optimal dual values. This procedure is repeated until no path can be found, and this is called path generation for which the details can be found in [29]. The problem of finding such a path is called pricing problem. Note that for any fragment of such a path, if it is a mirror path, its length should be smaller than $L$. For example, consider the path $(0,3,2,3,1)$ in Fig. 5 (b), the length of two fragments, i.e., mirror path $(0,3,2)$ and mirror path $(2,3,1)$, should be less than $L$.

To model the pricing problem, we define a set of ports for every node. Each port is connected to an arc attached to the 
node. The set of index of the ports for node $v$ is represented by $\rho_{v}$, the set of outgoing ports is represented by $\rho_{v}^{+}$and the set of incoming ports is represented by $\rho_{v}^{-}$. Let $\gamma_{a v}$, where $a \in \mathcal{A}$ and $v \in \mathcal{V}$, be the index of the port in node $v$ connecting arc $a$. For any arc $a$, the head node and tail node are represented by $g_{a} \in \mathcal{V}$ and $h_{a} \in \mathcal{V}$ respectively. The pricing problem is formulated in (15), while the variables are defined as follows.

$x_{a}$ binary variable, $x_{a}=1$ if arc $a$ is used; $x_{a}=0$ otherwise.

$u_{e}$ binary variable, $u_{e}=1$ if link $e$ is used; $u_{e}=0$ otherwise.

$y_{v i}^{+}$continuous variable, representing the transmitted distance when the signal is sent out from outgoing port $i \in \rho_{v}^{+}$of node $v \in \mathcal{V}$.

$y_{v i}^{-}$continuous variable, representing the transmitted distance when the signal arrives at incoming port $i \in \rho_{v}^{-}$ of node $v \in \mathcal{V}$.

$z_{v i j}$ binary variable, $z_{v i j}=1$ if the signal in incoming port $j \in \rho_{v}^{-}$is forwarded to outgoing port $i \in \rho_{v}^{+}$for a node $v \in \mathcal{V}$.

Minimize $c_{1} \sum_{v \in \mathcal{V}^{F}} \sum_{a \in \mathcal{A} \backslash \mathcal{A}^{O}} \delta_{a v} x_{a}+\sum_{e \in \mathcal{E}^{F} \cup \mathcal{E}^{M}} \pi_{e}^{*} u_{e}$ $+\frac{c_{3}}{2} \sum_{a \in \mathcal{A}^{M}} \sum_{v \in \mathcal{V}^{M}} \delta_{a v} x_{a}$

$\sum_{a \in \mathcal{A}} \delta_{a v}^{+} x_{a}-\sum_{a \in \mathcal{A}} \delta_{a v}^{-} x_{a}=\left\{\begin{array}{l}1, v=s \\ -1, v=t \\ 0, \text { otherwise }\end{array}\right.$

$y_{v i}^{+}=0, i \in \rho_{v}^{+}, v \in \mathcal{V}^{F}$

$y_{h_{a} i}^{-}=y_{g_{a} j}^{+}+l_{a} x_{a}, i=\gamma_{a h_{a}}, j=\gamma_{a g_{a}}, a \in \mathcal{A}$

$y_{v i}^{+}-y_{v j}^{-} \leq L\left(1-z_{v i j}\right), i \in \rho_{v}^{+}, j \in \rho_{v}^{-}, v \in \mathcal{V}^{M}$

$y_{v i}^{+}-y_{v j}^{-} \geq L\left(z_{v i j}-1\right), i \in \rho_{v}^{+}, j \in \rho_{v}^{-}, v \in \mathcal{V}^{M}$

$0 \leq y_{v i}^{+} \leq L, 0 \leq y_{v j}^{-} \leq L, i \in \rho_{v}^{+}, j \in \rho_{v}^{-}, v \in \mathcal{V}$

$z_{v \gamma_{a v} i} \leq x_{a}, i \in \rho_{v}^{-}, v=g_{a}, v \in \mathcal{V}^{M}, a \in \mathcal{A}^{M}$,

$z_{v i \gamma_{a v}} \leq x_{a}, i \in \rho_{v}^{+}, v=h_{a}, v \in \mathcal{V}^{M}, a \in \mathcal{A}^{M}$

$\sum_{i \in \rho_{v}^{+}} z_{v i j}=1, j \in \rho_{v}^{-}, v \in \mathcal{V}^{M}$

$\sum_{j \in \rho_{v}^{-}} z_{v i j}=1, i \in \rho_{v}^{+}, v \in \mathcal{V}^{M}$

$x_{a} \leq u_{f(a)}, a \in \mathcal{A}$.

According to the rule of adding a new path described above, the objective of the pricing problem is expressed as (15a). Constraints (15b) are the flow conservation rule, which is used to find a path that connects the source $s$ and the destination $t$ of a commodity. When an optical signal is transmitted by an FSO node, the transmitted distance is set to be 0 , this is stated by the constraint $(15 \mathrm{c})$. When an optical signal is transmitted along an arc $a$, from the outgoing port $\gamma_{a g_{a}}$ of node $g_{a}$ to the incoming port $\gamma_{a h_{a}}$ of node $h_{a}$, the length of the $\operatorname{arc}\left(l_{a}\right)$ is accumulated. This is expressed by constraint (15d). When an optical signal arrives at a mirror node $v \in \mathcal{V}^{M}$, it will be forwarded from an incoming port to an outgoing port without changing the transmitted distance. This is assured by constraints (15e) and (15f). These constraints are equivalent to $\left|y_{v i}^{+}-y_{v j}^{-}\right| \leq L\left(1-z_{v i j}\right)$. If $z_{v i j}=1$, we have $y_{v i}^{+}=y_{v j}^{-}$. Otherwise, i.e., $z_{v i j}=0$, the inequalities hold trivially. Note that the maximum transmitted distance for an optical signal arriving at a node or transmitted from a node should be smaller than $L$, which is assured by constraint $(15 \mathrm{~g})$. In this way, the constraint for each mirror path is indicated. Further, constraint (15h) ensures that, if a mirror arc $a$ is not installed, and the head node $g_{a}$ is a mirror node, any signal from its incoming port $i$ should not be forwarded to its outgoing port $\gamma_{a g_{a}}$. Similarly, constraint (15i) guarantees that for the tail node of mirror arc $a$. Constraints $(15 \mathrm{j})$ and $(15 \mathrm{k})$ guarantee that signals in an incoming port must be forwarded to one outgoing port and vice visa. Finally, constraint (15l) assures that a link is installed if any corresponding arc is used. After solving the price problem, we can obtain $\Lambda_{e p}$ and $\Theta_{a p}$ for current path $p$, based on the optimal value of $u_{e}$ and $x_{a}$ respectively.

In order to solve the master problem, an initial feasible set of paths must be provided. This set can be obtained by solving model (16) through path generation. Suppose $\lambda, \pi_{e}, e \in$ $\mathcal{E}^{F} \cup \mathcal{E}^{M}$ are dual variables corresponding to (16b) and (16c) respectively. The optimum of this model will be reached if there are not paths such that $\sum_{e \in \mathcal{E}^{F} \cup \mathcal{E}^{M}} \Lambda_{e p} \pi_{e}^{*} \leq \lambda^{*}\left(\lambda^{*}, \pi_{e}^{*}\right.$ are optimal dual variables). In fact, we can stop the procedure when $Z=0$ (even if the optimum may not be reached). If in the optimum $Z \neq 0$, there are not $K$ link-disjoint paths for the considered commodity.

$$
\begin{aligned}
\operatorname{Minimize} & Z \\
& \sum_{p \in \mathcal{P}} X_{p}=K+Z \\
& \sum_{p \in \mathcal{P}} \Lambda_{e p} X_{p} \leq 1, e \in \mathcal{E}^{F} \cup \mathcal{E}^{M} \\
& X, Y, Z-\text { continuous. }
\end{aligned}
$$

Step 3. When the linear relaxation of the model (14) is solved, a feasibility check should be carried out to assure that there are $K$ link-disjoint paths. A easy way is solving the equivalent binary problem of (16). If the model is infeasible, then we have to solve the CRBND for the current commodity in order to obtain feasible paths. After computing paths for current commodity, we obtain the deployed links $\left\{e \in \mathcal{A}: U_{e}=1\right\}$, mirrors nodes $\left\{v \in \mathcal{V}^{M}: Y_{v}=1\right\}$ and the set of paths $\mathcal{P}$. For calculating paths of subsequent commodities, the deployed links and nodes will not be included in the cost.

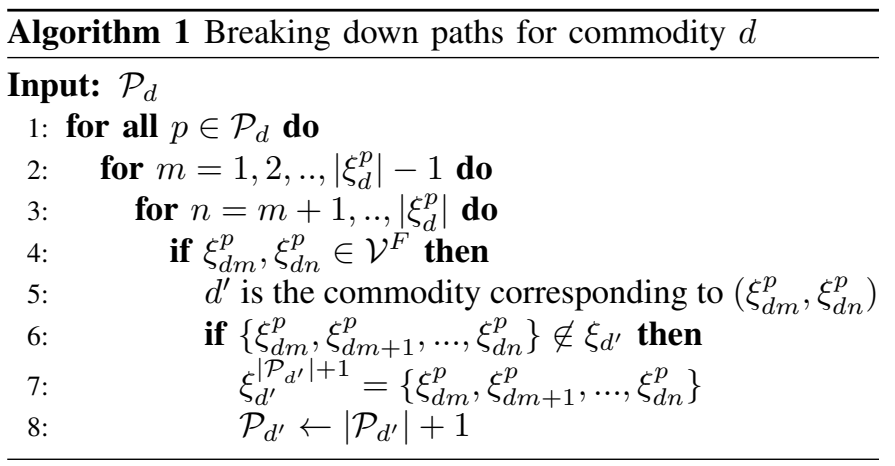

To accelerate the path generation for subsequent commodities, we break down the obtained paths of the current commodity to find more paths which can be used for subsequent commodities. For current commodity $d$, the set of generated paths is denoted by $\mathcal{P}_{d}$. Let $\xi_{d}^{p}$ be a set of nodes in path $p \in \mathcal{P}_{d}$, $\xi_{d n}^{p}$ be the $n$-th node in path $p$, and $\xi_{d}=\left\{\xi_{d}^{p}, p \in \mathcal{P}_{d}\right\}$. The 
procedure of breaking down paths is shown in Algorithm 1.

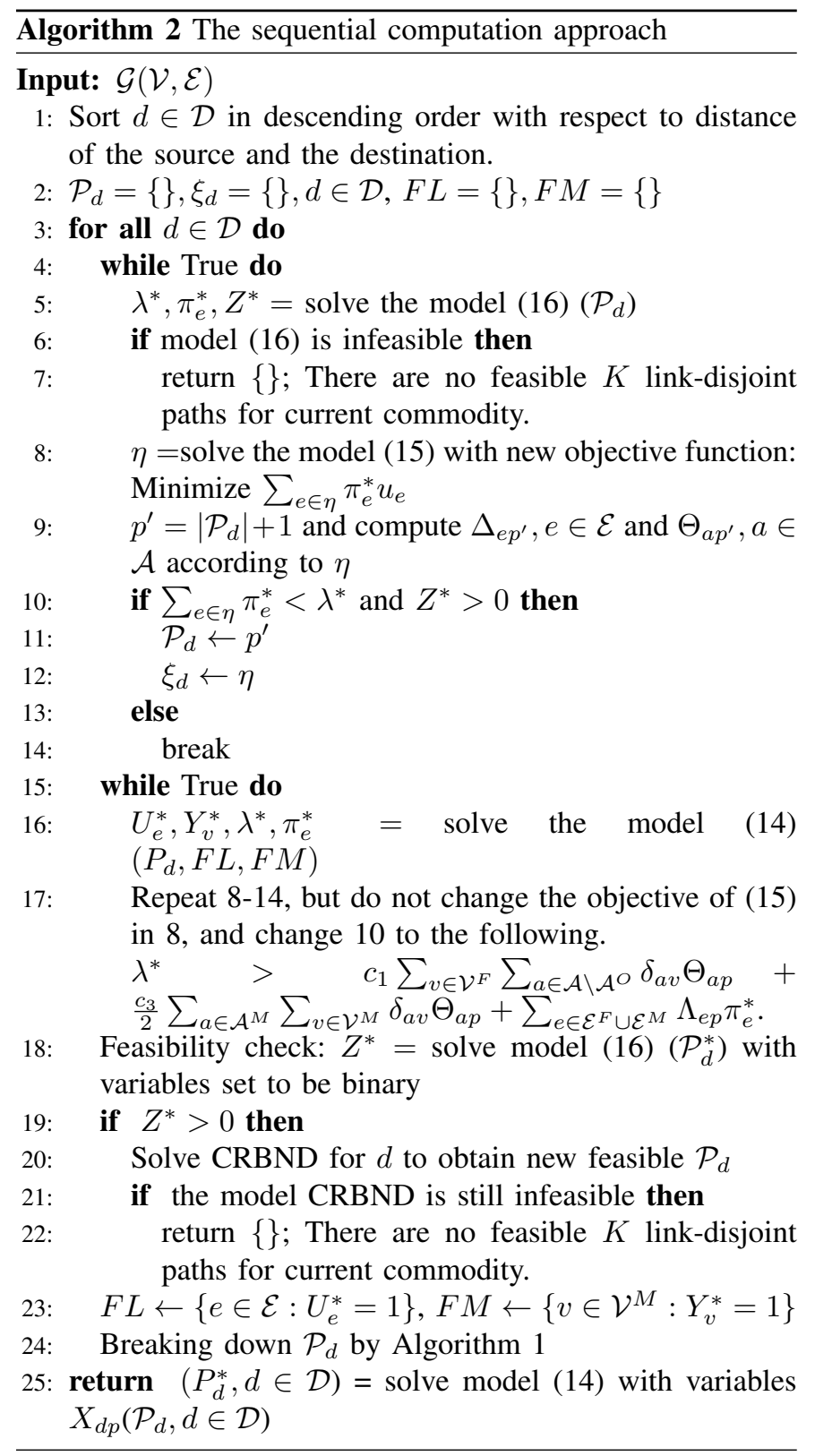

Step 4. After obtaining paths for all commodities, we consider to optimize the deployment cost and the network reliability based on all paths by solving a mixed integer programming model derived from model (14). We introduce binary variables $X_{d p}$ to replace $X_{p}$, indicating if the path $p$ is deployed by commodity $d$. The constant $\Lambda_{e p}$ is also replaced by $\Lambda_{e d p}$, representing whether link $e$ is included in path $p$ of commodity $d$. Constraint (14c) is changed to $\sum_{p \in \mathcal{P}} \Lambda_{e d p} X_{d p} \leq U_{e}, e \in \mathcal{E}^{F} \cup \mathcal{E}^{M}, d \in \mathcal{D}$ and adding constraints $\sum_{d \in \mathcal{D}} \sum_{p \in \mathcal{P}} \Lambda_{e d p} X_{d p} \geq U_{e}, e \in \mathcal{E}^{F} \cup \mathcal{E}^{M}$ to ensure link $e$ will not be established when there are no flows on it.

In summary, the sequential computation approach is presented in Algorithm 2, where $F L$ is the set of deployed links, $F M$ is the set of used mirror nodes, and $\eta$ represents a newly generated path.

Using the Algorithm 2, we can find optimal $K$ link-disjoint routing paths for all commodities. However, the number of FSO transceivers and mirrors is not explicitly given. It is not correct to count them on each path and then sum them all together. Since some FSO transceivers and mirrors can serve different commodities. Therefore, we propose the Algorithm 3 to count FSO transceivers and mirrors based on obtained paths. Let $F$ and $M$ denote the number of FSO transceivers and the number of mirrors respectively. For each path of a commodity, following the order of links in the path, we take a mirror path, count FSO transceivers and mirrors and then select the next one. Note that each mirror path has its own FSO transceivers and mirrors. To avoid duplicated counting FSO transceivers and mirrors for different commodities, each mirror path is counted only once.

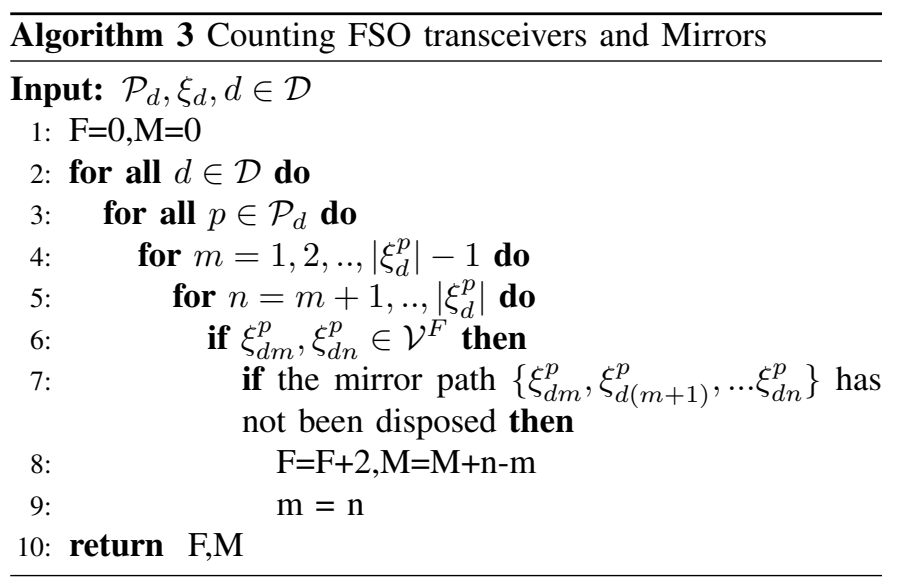

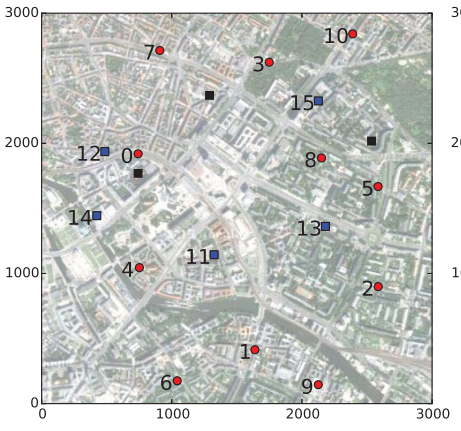

(a) R1

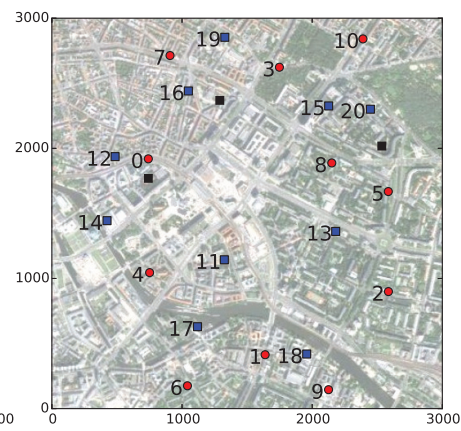

(b) R2
Fig. 6: Two scenarios of base stations in Berlin.

\section{NUMERICAL RESULTS}

In this section we provide numerical results regarding optimal topologies and optimal deployment of network devices under certain realistic scenarios. The impact of different parameters on the design of the network is also analyzed. Moreover, the efficiency of Algorithm 2 is compared with the exact model under some randomly generated networks. We set $I_{t h} / I_{0}=0.8$ according to [26]; an FSO link or a mirror link can be established if its length is smaller than $1400 \mathrm{~m}$. The objective includes two parts, i.e., the deployment cost and the total reliability. The coefficients for the deployment cost is set as $c_{1}: c_{2}: c_{3}=4: 2: 1$. The coefficient 


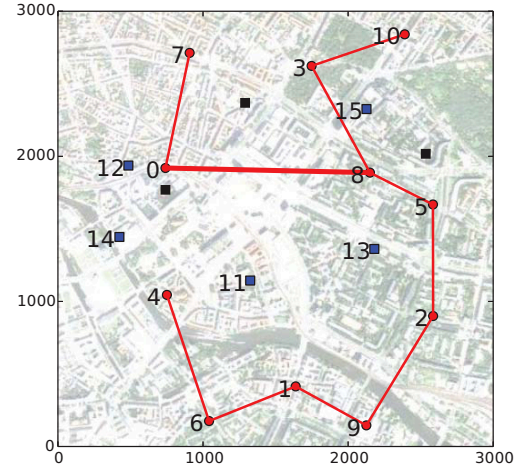

(a) 1-connected

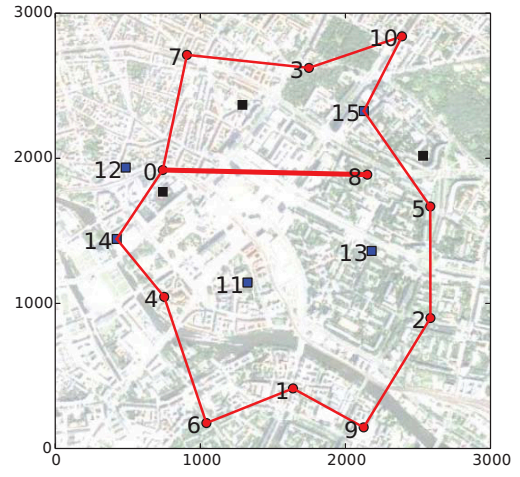

(b) 2-connected

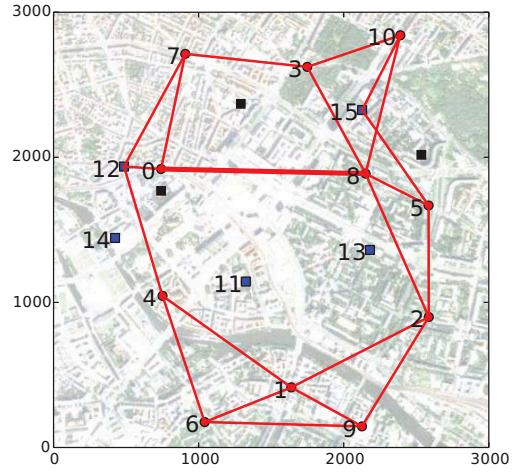

(c) 3-connected

Fig. 7: Optimal topologies with different connectivity for R1.

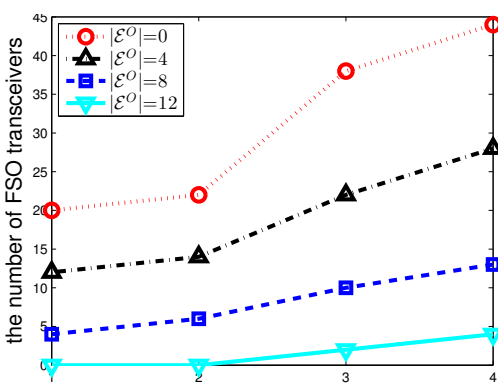

(a) FSO transceivers

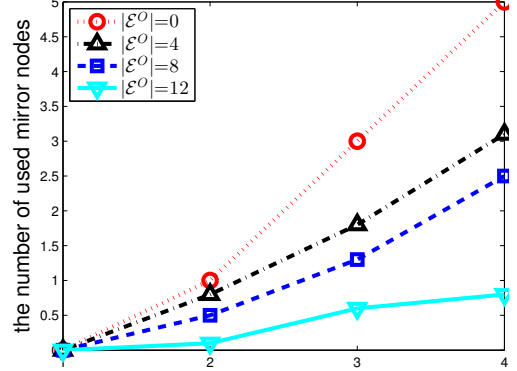

(b) Mirror Stations

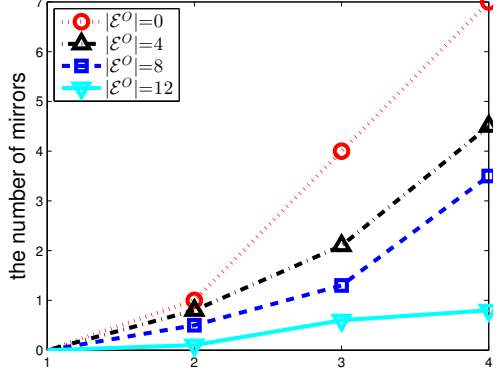

(c) Mirrors

Fig. 8: The number of FSO transceivers, Mirror Nodes, Mirrors v.s. Optical Fibers and $K$.

of the reliability is $c_{4}$ which will be adjusted to analyze the trade-off between the two type of sub-objectives. The Gurobi solver [37] is used for solving integer and linear programming problems. All the computations were executed on a Windows $\mathrm{XP}$ computer equipped with a dual core Intel 2.53 GHZ CPU and 1.93GB RAM.

The realistic network is a snapshot of the district around Alexanderplatz of Berlin, and includes 11 base stations in an area of $3000 \times 3000 \mathrm{~m}^{2}$, which are provided by the EU MOMENTUM project [38]. Fig. 6 shows a map with different number of mirror nodes; the red circles are base stations, the blue squares are the candidate locations, building roofs, for deploying mirrors. The black unnumbered squares represent high buildings which are treated as obstacles. Regarding the random networks, we randomly generate the same number of FSO nodes and mirror nodes in a square area of $3000 \times 3000 \mathrm{~m}^{2}$.

\section{A. A case study}

Utilizing the CRBND model, we compute optimal solutions for R1, and then evaluate the impact of different parameters on optimal solutions. This case is studied from the cost perspective, i.e., $c_{1}: c_{4}=10: 1$.

In $\mathrm{R} 1$, there is an fiber link between nodes 0 and 8 represented by a thick line. The optimal topologies for $K=1,2,3$ are presented in Fig. 7. Fig. 7(a) shows a 1-connected topology with 16 FSO transceivers and 0 mirrors. Fig. 7(b) shows a 2-connected topology consisting of 20 FSO transceivers, 2 mirrors and 2 mirror stations. Nodes 14 and 15 have mirrors to connect the FSO nodes that are not in the line of sight. Note that paths using the fiber link between nodes 0 and 8 are linkdisjoint. Fig. 7(c) shows a 3-connected graph with 38 FSO transceivers and 3 mirrors. There are two mirrors deployed in node 12 . One is used to connect nodes 0 and 7 , the other one is used to connect nodes 0 and 4. Mirror node 14 is not used in order to reduce the cost of leasing mirror nodes. Note that the link $\{0,12\}$ corresponds to 2 FSO transceivers in node 0 .

In R2, we study the impact of $K$ and the number of available optical fibers on the optimal solution. We consider four cases regarding the number of optical fibers: 0,4,8,12. For each case, we test 10 instances and then take the average over the obtained results. The number of FSO transceivers, mirrors, leased mirror nodes for $K=1,2,3,4$ are plotted in Fig. 8. In Fig. 8(a), we can see that as $K$ increases, the number of FSO transceivers increases dramatically, which is more significant than the increase of the number of mirrors in Fig. 8(c) and the number of mirror nodes in Fig. 8(b). This is because an FSO link needs two FSO transceivers and some mirror links also need FSO transceivers. As illustrated in Fig. 8(a), when more optical fibers are available, the number of FSO transceivers grows slowly with the increase of $K$. By adding 12 optical fibers, the number of FSO transceivers for $K=4$ decreases by 40 compared to the case without optical fibers, thus, around $90 \%$ of FSO transceivers are saved. The increasing trend for the number of mirrors in Fig. 8(c) is similar to the trend for the number of used mirror nodes in Fig. 8(b). However, the number of used mirror nodes increases slower than the number of mirrors, since a mirror node can accommodate more than one mirrors and the deployment of optical fibers slows 
TABLE II: Comparison of the result and the running time.

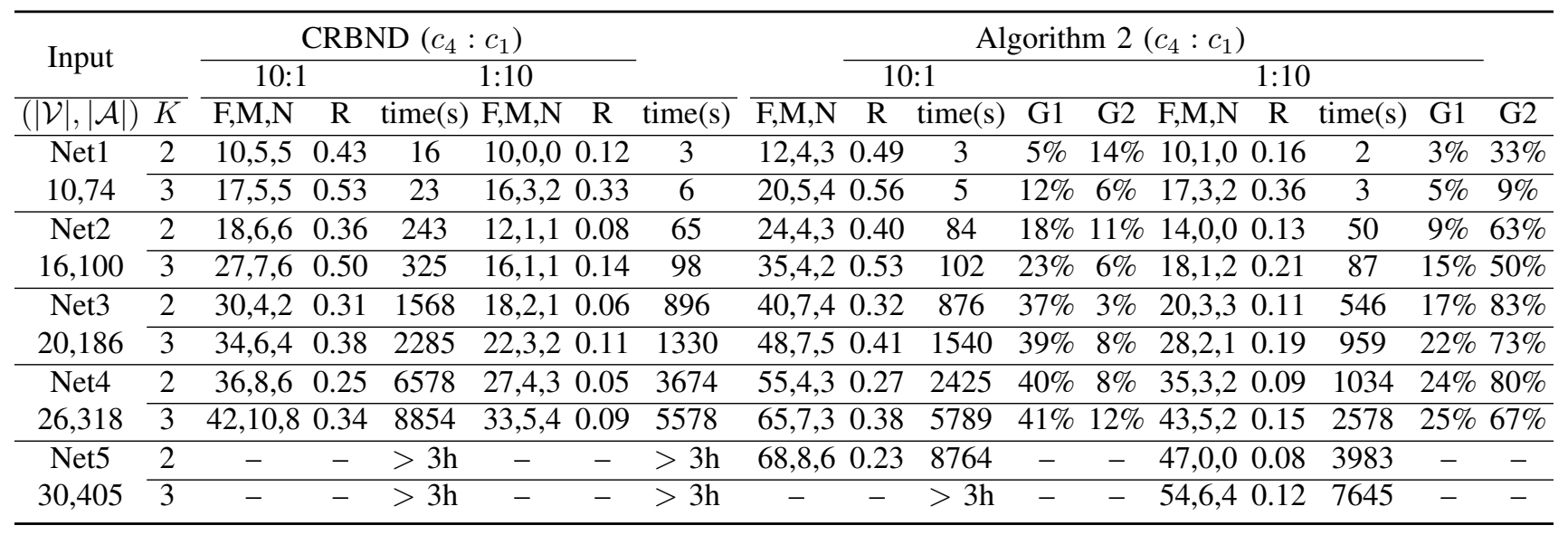

TABLE III: Complexity comparison of solution approaches.

\begin{tabular}{cccccc}
\hline \multirow{2}{*}{ Input } & \multirow{2}{*}{ Exhaustive Search } & \multirow{2}{*}{ CRBND } & \multicolumn{3}{c}{ Algorithm 2 } \\
\cline { 4 - 6 } & & & master problem & pricing problem & iterations \\
\hline Net1 & $\left(2^{8} \times\left(2^{8}-1\right)\right)^{10}$ & $4.5 \times 10^{4}$ & 54 & 921 & 4 \\
Net2 & $\left(2^{14} \times\left(2^{14}-1\right)\right)^{28}$ & $1.8 \times 10^{5}$ & 76 & 1446 & 7 \\
Net3 & $\left(2^{18} \times\left(2^{18}-1\right)\right)^{45}$ & $9.7 \times 10^{5}$ & 126 & 4199 & 9 \\
Net4 & $\left(2^{24} \times\left(2^{24}-1\right)\right)^{73}$ & $4.8 \times 10^{6}$ & 200 & 9863 & 10 \\
Net5 & $\left(2^{28} \times\left(2^{28}-1\right)\right)^{105}$ & $7.4 \times 10^{6}$ & 253 & 13191 & 12 \\
\hline
\end{tabular}

down the increasing trend. The network reliability is improved when $K$ increases and more fiber links are added, which is a reasonably expected outcome.

Furthermore, as we can see, to achieve 2-connected topology for R2 without optical fibers, our optimized solution needs 22 FSO transceivers and 1 mirror while without optimization 146 FSO transceivers and 22 mirrors are needed if we consider the input network as a solution. This illustrates the necessity of our proposed model.

\section{B. Algorithm comparison}

In this section we compare the sequential computation approach (Algorithm 2) with the exact optimization model, CRBND, through a large number of experiments.

Table II shows optimal results and running times for the two methods under two settings, i.e., $c_{4}: c_{1}=10: 1$ and $c_{4}: c_{1}=1: 10$. The optimal results include the number of FSO transceivers $(F)$, mirror nodes $(N)$, mirrors $(M)$ and the normalized reliability $(R)$. The normalized reliability is the total reliability of FSO and mirror links in the optimal solution divided by the total reliability of all potential FSO and mirror links. The entry " $>3 \mathrm{~h}$ " in the table denotes that the model or the algorithm cannot produce an optimal solution in 3 hours, and "-" is inserted to the corresponding results. We test a large variety of networks with different number of nodes. For each input network, we select randomly $10 \%$ of links to be nonline-of-sight links, and select $5 \%$ pairs of FSO nodes between which optical fibers are used. All results are averaged over 10 instances.

From Table II, we can see that the optimal solution corresponding to $c_{4}: c_{1}=10: 1$ has higher reliability than that for the setting $c_{4}: c_{1}=1: 10$. However, the former one has higher cost in terms of the number of FSO transceivers, mirrors and leased mirror nodes. Under the same setting, i.e., the same $c_{4}: c_{1}$, the proposed sequential computation approach always obtains a solution with higher reliability, since it uses more links in the solution than CRBND, and thus needs higher deployment cost. Additionally, we illustrate the gap for the cost (G1) and the gap for the reliability (G2) of Algorithm 2. G1 is defined as: (the cost obtained by Algorithm 2 - the cost obtained by CRBND) / the cost obtained by CRBND $\times$ $100 \%$. The cost for any of the two methods is computed by $c_{1} \times F+c_{2} \times N+c_{3} \times M$. G2 is defined similarly. As we can see in the table, when the weight of the reliability is higher, the gap of the reliability between the two methods is smaller but the gap of the cost becomes bigger. Similarly, when the weight of cost is higher, the gap of cost becomes smaller but the gap of the reliability becomes bigger.

Notably, the running time of the model CRBND for $c_{4}$ : $c_{1}=10: 1$ is much higher than that for $c_{4}: c_{1}=1: 10$ while the running times for Algorithm 2 for two settings have relative small gap. This indicates that when the weight of the reliability increases, the running time of the model CRBND increases much faster than the sequential computation approach. For small input networks, say 10 nodes, the model CRBND can compute optimal solutions quickly. However, when the network size increases, even to a medium size, e.g., 26 nodes, the model CRBND will be quite time consuming. Thus, we need to use the proposed sequential computation approach. For the impact of $K$ on the two methods, bigger $K$ takes longer running time for both methods. Even only increasing by one for $K$, the running times for both methods 
increase a lot.

Furthermore, we illustrate that the exhaustive search method is not practical for solving considered problems. Let $P_{d}$ be the set of all possible paths for each commodity $d \in \mathcal{D}$, i.e., every pair of FSO nodes. There are $2^{|\mathcal{V}|-2}$ possible paths in the set. To find a $K$-connected graph with the objective considered in question, an exhaustive search would require to generate all possible subsets of $K$ paths for each commodity and then consider all combinations of such subsets over the commodities. Thus, the total number of combinations for this exhaustive search is $\prod_{d \in \mathcal{D}}\left(\begin{array}{c}P_{d} \\ K\end{array}\right)=\left(\begin{array}{c}2^{|\mathcal{V}|-2} \\ K\end{array}\right)^{\mathcal{V}^{F}\left(\mathcal{V}^{F}-1\right) / 2}$, and the time complexity for computing the objective for each of them amounts to $\mathcal{O}\left(K\left(\mathcal{V}^{F}\right)^{2} \mathcal{V}^{2}\right)$ (according to Algorithm 3). In Table III, we compute the number of operations for this exhaustive search for networks tested in Table II with $K=2$. As we can see, for Net3, to find a 2-connected subgraph, the complexity of exhaustive search is more than $\left(2^{18}\left(2^{18}-1\right)\right)^{45}$, which is clearly beyond what can be practically dealt with.

Both the CRBND model and the proposed computation approach are not polynomial-time methods, and no exact expressions can be found for their time complexities. However, the sequential computation approach utilizes the idea of problem decomposition, which is a natural and effective way to tackle large mathematical programming problems. We compare the two methods in terms of the total number of binary and integer variables. The CRBND model has $K\left|\mathcal{V}^{F}\right|^{2}\left(3\left|\mathcal{E}^{F}\right|+|\mathcal{E}|+3\left|\mathcal{E}^{M}\right|\left|\mathcal{E}^{F}\right|\right)+2\left|\mathcal{E}^{F}\right|+|\mathcal{E}|+|\mathcal{V}|$ noncontinuous variables. For the sequential computation approach, we find $K$ link-disjoint paths commodity by commodity. For each commodity, the original problem is relaxed and decomposed to a master problem and a pricing problem, each with a considerably smaller number of variables. The master problem has $|\mathcal{E}|+|\mathcal{V}|+|\mathcal{P}|$ variables, and the pricing problem has $3|\mathcal{E}|+|\mathcal{V}| \rho_{\text {max }}^{2}$ (where $\rho_{\max }$ is the maximum node degree in the undirected tested network). The number of paths $|\mathcal{P}|$ is set to zero at the beginning for solving the master problem and increases after each iteration for generating paths. Typically, when the final master problem is solved, $|\mathcal{P}|$ will not be large, which is discussed subsequently.

In Table III, for each input network, we estimate the number of non-continuous variables for CRBND and for the master problem and related pricing problem in Algorithm 2. Note that many master problems are needed to be solved for Algorithm 2, we only show in Table III the one with the most number of iterations, i.e, each iteration is to solve the pricing problem to find a path. As we can see, for all tested networks, the number of paths $|\mathcal{P}|$ for the master problem (the number of non-continuous variables $-\frac{1}{2}|\mathcal{A}|-|\mathcal{V}|$ ) is small. Taking Net4 as an example, the number of variables in CRBND is of the magnitude of $10^{6}$ - a number not easy to deal with using commercial solvers. By decomposition, the number of non-continuous variables are greatly reduced for each subproblem, which can be quickly solved. Moreover, the number of variables in CRBND increases dramatically with the size of the network.

At last, we study the benefits of using Algorithm 1 to accelerate Algorithm 2. The tested networks are randomly generated and the experiments are conducted under parameters $c_{4}: c_{1}=1: 1$ and $K=2$. For networks with the same number of nodes, 10 instances are tested and average results are presented. The running times for Algorithm 2 with Algorithm 1 (T1) and without Algorithm 1 (T2) are shown in Table IV. We see that Algorithm 1 helps a lot to reduce the running time of Algorithm 2.

TABLE IV: Comparison of the running time of Algorithm 2 with and without Algorithm 1.

\begin{tabular}{|c|c|c|c|c|c|}
\hline & 10 & 16 & 20 & 26 & 30 \\
\hline T1 (s) & 4 & 60 & 689 & 1123 & 4456 \\
\hline T2 (s) & 8 & 115 & 1764 & 3565 & $>3 \mathrm{~h}$ \\
\hline
\end{tabular}

\section{CONCLUSION}

We have studied optimization formulation and solution approaches for designing FSO networks, taking into account cost and reliability as performance metrics. Network survivability is modelled by means of the $K$-connectivity requirement between FSO nodes. From an optimization viewpoint, the problem represents an unconventional setup within the domain of graph optimization, because of the possibility of using mirrors with distance consideration of such connections. Integer programming models based on the notions of network flow and path generation have been developed. The latter has been utilized to derive a sequential algorithm that, by the performance results, enables near-optimal solutions with much smaller computational effort in comparison to applying integer programming to the flow-based model. Moreover, the results illustrate the trade-off between cost and reliability, in particular for large-scale scenarios. Thus the study provides new insights into deploying cost-effective and high-performance networks with the FSO technology.

Furthermore, the presented model and method can be used for designing networks based on any up-to-date outdoor FSO links. Even though the numerical results are obtained using the log-normal channel model that characterizes atmospheric fading, our optimization framework is not limited to a particular channel type. As the optimization model and the algorithm do not make any restriction on how the input data is generated, other types of FSO channel models, for example the GammaGamma model, can be used as the underlying input generator. For the same reason, within the same optimization framework, the channel model between an FSO node and mirror could be different from that for the FSO nodes. This paper proposes a novel idea to use mirrors to connect FSO transceivers that are not in line of sight. Mirrors can be treated as relays in a network without the ability of amplifying. Although there has been some work analysing the performance of FSO links with relays (not mirrors), see for example [39], it would be interesting to investigate the impact of using mirrors on the performance of FSO links.

\section{REFERENCES}

[1] R. Ford, C. Kim, and S. Rangan, "Opportunistic third-party backhaul for cellular wireless networks," ArXiv e-prints, 2013. 
[2] O. Tipmongkolsilp, S. Zaghloul, and A. Jukan, "The evolution of cellular backhaul technologies: current issues and future trends," IEEE Communications Surveys Tutorials, vol. 13, no. 1, pp. 97-113, 2011.

[3] T. Frey, "The effects of the atmosphere and weather on the performance of a mm-wave communication link," Applied Microwave and Wireless, pp. 76-80, 1999.

[4] S. Chia, M. Gasparroni, and P. Brick, "The next challenge for cellular networks: backhaul," IEEE Microwave Magazine, vol. 10, no. 5, pp. 54-66, 2009.

[5] V. Chan, "Free-space optical communications," IEEE/OSA Journal of Lightwave Technology, vol. 24, no. 12, pp. 4750-4762, 2006.

[6] H. H. Refai, J. J. Sluss, and M. Atiquzzaman, "Comparative study of the performance of analog fiber optic links versus free-space optical links," Optical Engineering, vol. 45, no. 2, 2006.

[7] Z. Ghassemlooy, W. Popoola, and S. Rajbhandari, Optical Wireless Communications: System and Channel Modelling with MATLAB. CRC Press, 2012.

[8] Z. Xu and B. Sadler, "Ultraviolet communications: Potential and stateof-the-art," IEEE Communications Magazine, vol. 46, no. 5, pp. 67-73, 2008.

[9] T.-H. Ho, "Pointing, acquisition, and tracking systems for free-space optical communication links," Ph.D. dissertation, University of Maryland.

[10] T. Tidwell, J. Gregory, C. Chalfant, F. Orlando, and M. Leftwich, "Rapid acquisition, pointing and tracking optical system for free space optical communications," 2012, US Patent 8,160,452.

[11] F. Demers, H. Yanikomeroglu, and M. St-Hilaire, "A survey of opportunities for free space optics in next generation cellular networks," in the 9th Annual Communication Networks and Services Research Conference (CNSR), May 2011, pp. 210-216.

[12] M. R. Garey and D. S. Johnson, Computers and intractability: A guide to the theory of NP-completeness. American Mathematical Society, 1980.

[13] H. Kerivin and A. Mahjoub, "Design of survivable networks: A survey," Networks, vol. 46, no. 1, pp. 1-21, 2005.

[14] R. Khandekar, G. Kortsarz, and Z. Nutov, "On some network design problems with degree constraints," Journal of Computer and System Sciences, vol. 79, no. 5, pp. 725-736, 2013.

[15] F. Bendali, I. Diarrassouba, A. Mahjoub, and J. Mailfert, "The edgedisjoint 3-hop-constrained paths polytope," Discrete Optimization, vol. 7, no. 4, pp. 222-233, 2010

[16] M. Zotkiewicz, W. Ben-Ameur, and M. Pioro, "Finding failure-disjoint paths for path diversity protection in communication networks," IEEE Communications Letters, vol. 14, no. 8, pp. 776-778, 2010.

[17] Y. Wu, F. Wang, M. Thai, and Y. Li, "Constructing k-connected mdominating sets in wireless sensor networks," in IEEE Military Communications Conference, October 2007, pp. 1-7.

[18] Y. Wu and Y. Li, "Construction algorithms for k-connected $\mathrm{m}$ dominating sets in wireless sensor networks," in ACM International Symposium on Mobile Ad Hoc Networking and Computing (MobiHoc), May 2008, pp. 83-90.

[19] D. Li, J. Cao, M. Liu, and Y. Zheng, "K-connected target coverage problem in wireless sensor networks," in Combinatorial Optimization and Applications, ser. Lecture Notes in Computer Science, 2007, pp. 20-31.

[20] M. Younis, I. F. Senturk, K. Akkaya, S. Lee, and F. Senel, “Topology management techniques for tolerating node failures in wireless sensor networks: A survey," Computer Networks, vol. 58, no. 0, pp. 254-283, 2014.

[21] M. Khalighi and M. Uysal, "Survey on free space optical communication: A communication theory perspective," IEEE Communications Surveys Tutorials, 2014.

[22] J. Llorca, A. Desai, and S. Milner, "Obscuration minimization in dynamic free space optical networks through topology control," in IEEE MILCOM, October 2004, pp. 1247-1253.

[23] J. Zhuang, M. Casey, S. Milner, S. Gabriel, and G. Baecher, "Multiobjective optimization techniques in topology control of free space optical networks," in IEEE MILCOM, October 2004, pp. 430-435.

[24] X. Cao, "An integer linear programming approach for topology design in OWC networks," in IEEE GLOBECOM Workshops, November 2008 , pp. 1-5.

[25] A. Kashyap, K. Lee, M. Kalantari, S. Khuller, and M. Shayman, "Integrated topology control and routing in wireless optical mesh networks," Computer Networks, vol. 51, no. 15, pp. 4237-4251, 2007.

[26] I. Son and S. Mao, "Design and optimization of a tiered wireless access network," in IEEE INFOCOM, March 2010, pp. 1-9.
[27] H. Zhou, A. Babaei, S. Mao, and P. Agrawal, "Algebraic connectivity of degree constrained spanning trees for fso networks," in IEEE ICC, June 2013, pp. 5991-5996.

[28] I. Ouveysi, F. Shu, W. Chen, G. Shen, and M. Zukerman, "Topology and routing optimization for congestion minimization in optical wireless networks," Optical Switching and Networking, vol. 7, no. 3, pp. 95-107, 2010.

[29] M. Pióro and D. Medhi, Routing, Flow, and Capacity Design in Communication and Computer Networks. Morgan Kaufmann, 2004.

[30] I. K. Son, S. Mao, and S. K. Das, "On joint topology design and load balancing in free-space optical networks," Optical Switching and Networking, vol. 11, Part A, pp. 92-104, 2014.

[31] I. Cox, C., E. Ackerman, R. Helkey, and G. Betts, "Techniques and performance of intensity-modulation direct-detection analog optical links," IEEE Transactions on Microwave Theory and Techniques, vol. 45, no. 8 , pp. 1375-1383, 1997.

[32] S. Arnon, "Optimization of urban optical wireless communication systems," IEEE Transaction on Wireless. Communication, vol. 2, no. 4, pp. 626-629, 2003

[33] A. Farid and S. Hranilovic, "Outage capacity optimization for free-space optical links with pointing errors," Journal of Lightwave Technology, vol. 25 , no. 7 , pp. 1702-1710, 2007.

[34] N. Vaiopoulos, H. Sandalidis, and D. Varoutas, "WiMAX on FSO: outage probability analysis," IEEE Transactions on Communications, vol. 60 , no. 10 , pp. $2789-2795,2012$.

[35] J. C. Ricklin and F. M. Davidson, "Atmospheric turbulence effects on a partially coherent gaussian beam: implications for free-space laser communication," Journal of the Optical Society of American A, vol. 19, no. 9, pp. 1794-1802, 2002.

[36] X. Zhu and J. Kahn, "Free-space optical communication through atmospheric turbulence channels," IEEE Transactions on Communications, vol. 50, no. 8, pp. 1293-1300, 2002.

[37] "Gurobi Optimizer," http://www.gurobi.com.

[38] "Momentum project, 2003 (updated in 2005)," http://momentum.zib.de.

[39] M. Safari and M. Uysal, "Relay-assisted free-space optical communication," IEEE Transactions on Wireless Communications, vol. 7, no. 12, pp. 5441-5449, 2008.

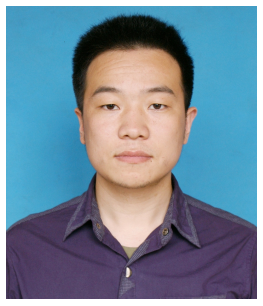

Yuan Li received his BSc degree and finished his master program in computer science and engineering in 2008 and 2010 respectively, both at National University of Defense Technology, China. He has obtained his PhD degree in the Department of Electrical and Information Technology at Lund University, Sweden, in 2015. At present he is an Assistant Professor at School of Computer, National University of Defense Technology. His research interests are in network modeling, algorithm design, integer programming and other combinatorial methods with applications in communication networks.

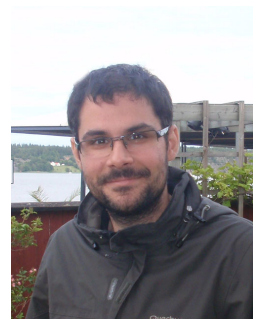

Nikolaos Pappas received his BSc degree in Computer Science from University of Crete, Greece in 2005, and MSc degree in Computer Science from University of Crete, Greece, in 2007. He also obtained his BSc degree in Mathematics from University of Crete, Greece in 2012. He holds a PhD degree from the Department of Computer Science of the University of Crete, Greece in 2012. From 2005 to 2012 , he was a graduate research assistant with the Telecommunications and Networks Laboratory of the Institute of Computer Science of the Foundation for Research and Technology, Hellas (FORTH) and he has been a visiting scholar of the Institute of Systems Research at the University of Maryland in College Park. From September 2012 to March 2014, he was a postdoctoral researcher at the Department of Telecommunications, at Supélec, France. Since March 2014 he is at the University of Linköping at Norrköping Campus as a Marie Curie Fellow (IAPP). His main research interests are in the field of wireless communication networks with emphasis on the stability analysis, energy harvesting networks, network-level cooperation, network coding and stochastic geometry. He is an Editor for the IEEE Communications Letters. 


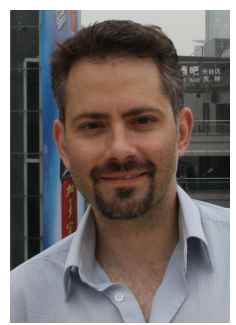

Vangelis Angelakis (S'05-M'09) holds an MSc('04) and a $\mathrm{PhD}(' 08)$ in Computer Science from the University of Crete, Greece. Presently, he is an Assistant Professor in Mobile Telecommunications at the Department of Science and Technology, Linkping University. Dr Angelakis has been a research assistant at the Institute of Computer Science (ICS) of the Foundation for Research And TechnologyHellas (FORTH), Greece since 2001. In 2005 he was a visiting research associate at the Institute for Systems Research of the University of Maryland in College Park, USA. From 2008 he was a postdoctoral researcher with the Telecommunications and Networks laboratory of FORTH-ICS, until 2009 when he joined Linköping University. Since then he has been a visiting researcher in the UK, China, and Greece. He is an associate editor of the Journal of Communications and Networks and has received support from national funding bodies in Greece and Sweden and the EU within FP7 and $\mathrm{H} 2020$. His current research interests focus on resource allocation in wireless networks and their applications.

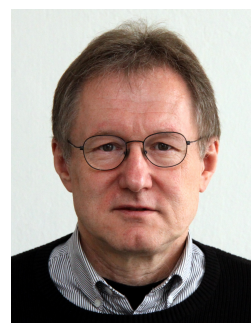

Michał Pióro is a professor and head of Computer Networks and Switching Division, Institute of Telecommunications, Warsaw University of Technology, Poland, and a professor at Lund University, Sweden. He received a Ph.D. degree in telecommunications in 1979 and the D.Sc. degree in 1990, both from the Warsaw University of Technology. In 2002 he received a Polish State Professorship. His research interests concentrate on modeling, design and performance evaluation of communication and computer networks. He is an author of four books (including well known monograph: M. Piro and D. Medhi Routing, Flow, and Capacity Design in Communication and Computer Networks, MorganKaufmann, USA, 2004) and around 150 technical papers presented in communications and OR journals and conference proceedings. He has led many national and international research projects in the field for telecom industry and EC.

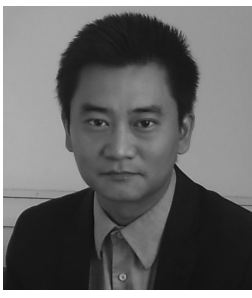

Di Yuan received his MSc degree in Computer Science and Engineering, and $\mathrm{PhD}$ degree in operations research at Linköping University, Sweden, in 1996 and 2001, respectively. At present he is full professor in telecommunications at the Department of Science and Technology, Linköping University, and head of a group in mobile telecommunications. Di Yuan has been part time with Ericsson Research, Sweden. He is a co-recipient of IEEE ICC 12 Best Paper Award. In 2015 he is visiting professor at the Institute for Park, MD, USA. Systems Research, University of Maryland, College 